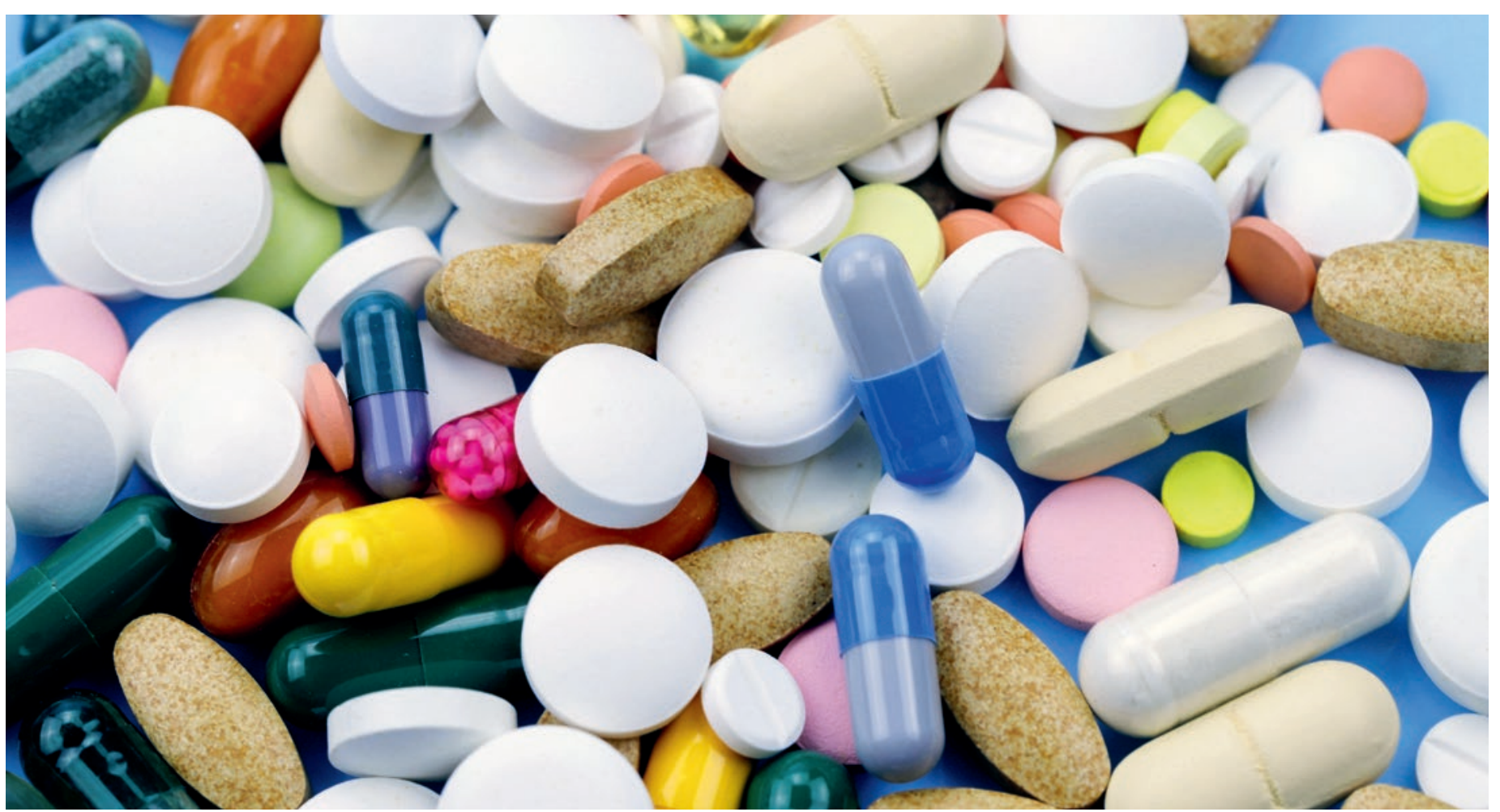

\title{
Quels sont les enseignements de la crise du coronavirus?
}

\section{Jürg Barbena, Thomas Cerny ${ }^{b}$}

a Professeur de pneumologie pédiatrique, Ostschweizer Kinderspital St-Gall; b Professeur émérite d’oncologie, président de la Recherche suisse contre le cancer

Avant la crise du coronavirus, les quotidiens titraient souvent sur la hausse des coûts du système de santé et la manière de les réduire efficacement. La justification des prix pour les traitements innovants se chiffrant en millions est également un sujet. La pandémie de coronavirus a mis les soins de santé dans la ligne de mire des médias et nous a démontré la valeur d'un système de santé qui fonctionne bien, même s'il a son prix.

Nous nous dirigeons désormais vers la «nouvelle normalité», et il est extrêmement indiqué de s'interroger à nouveau sur les coûts du système de santé. Par ailleurs, la question de savoir s'il est encore défendable du point de vue éthique de faire des milliards de profits dans le système de santé aux frais de personnes malades se pose également. La santé est le bien le plus précieux que nous ayons et, pour la conserver, nombreux sont ceux qui acceptent de dépenser beaucoup d'argent. De plus, le secteur global de la santé connaît une rapide expansion et s'avère très lucratif grâce aux marges bénéficiaires extrêmes dans toutes les catégories.

\section{Système de santé ou industrie de la santé?}

Giovanni Maio, spécialiste de l'éthique médicale, a formulé de façon très pertinente les conséquences de l'économisation du secteur de la santé dans son livre «Geschäftsmodell Gesundheit - Wie der Markt die Heilkunst abschafft (Modèle commercial de la santé ou comment le marché détruit l'art de guérir)»: «Un système de santé est un élément du système social de notre société. Une partie de notre richesse est investie dans la santé publique, pour le bien de tous. Une industrie de la santé, en revanche, fait partie du système économique. 
Les détenteurs de capitaux investissent dans cette industrie de la santé, et en attendent un rendement, pour le bien d'une minorité. Ces deux systèmes ne sont pas compatibles, car leurs objectifs sont fondamentalement contradictoires.» De nos jours, nombre de grands groupes cherchent à maximiser les profits, ce qui est absolument incompatible avec un système de santé solidaire.

\section{Le rôle de l'industrie pharmaceutique}

Depuis le début des années 80 , le marché global de la pharmacie n'a cessé de croître pour atteindre un volume de plus de 1000 milliards de dollars US par an, dont la moitié aux États-Unis. À elles seules, les dix premières firmes pharmaceutiques réalisent les deux tiers du chiffre d'affaires global (www.pmlive.com). Depuis l'ère Reagan, les grands groupes pharmaceutiques mettent en œuvre le modèle «blockbuster» (médicaments affichant des chiffres d'affaires annuels supérieurs à un milliard de dollars), avec maximisation des profits en respectant ou non les limites légales, des marges bénéficiaires supérieures à $20 \%$ étant la norme [1, 2]. En 2003, les bénéfices de l'industrie pharmaceutique dépassaient encore les bénéfices cumulés de tous les secteurs listés par Forbes. La belle époque des modèles blockbuster a toutefois une échéance, car on trouve désormais sur le marché de nombreux médicaments génériques contre des maladies très courantes comme l'hypertonie, l'asthme, la dépression ou les tumeurs. De plus, les brevets de nombreux médicaments à succès arrivent à leur terme et les génériques sont devenus nettement meilleur marché.

Dans l'industrie pharmaceutique, le nouveau mot magique, c'est désormais «la médecine personnalisée» [1]. Le développement des "orphan drugs», c'est-à-dire des médicaments contre les maladies orphelines comme

\section{Quel est le rôle des brevets?}

Les brevets ne sont ni une bonne chose, ni une mauvaise chose. Ils peuvent contribuer aux bénéfices d'une société, mais également lui nuire et nuire à son économie. Ils ne sont utiles que lorsque l'intérêt public de la collectivité prime. C'est en effet le seul moyen de justifier politiquement que l'État octroie un monopole à des individus ou des entreprises qui peuvent en faire une activité ne subissant aucune concurrence. De nos jours, on abuse souvent des brevets pour maximiser les profits, sans qu'il y ait utilité correspondante. De plus, les litiges portant sur des brevets entravent l'innovation et le développement. La mise sur le marché de génériques et biosimilaires s'en trouve retardée, voire empêchée.

Warum Patente in der Onkologie heute schädlich sind oder "It's the economy, stupid!» Bulletin suisse du cancer 2020;202:114-7. la mucoviscidose, amyotrophie spinale et de nombreuses tumeurs, a ouvert un nouveau marché d'avenir prometteur pour l'industrie pharmaceutique. Des incitations légales telles que la prolongation de la protection du brevet ont rendu intéressante la recherche sur les "orphan drugs». En conséquence, aujourd'hui, on réclame des sommes astronomiques se chiffrant en millions pour de tels médicaments.

\section{Rémunération de coûts de développe- ment élevés?}

S'agissant de la tarification, les représentants de l'industrie pharmaceutique argumentent qu'en raison des coûts de développement importants, des prix élevés se justifient. Toutefois, les instituts de recherche d'universités font de nombreuses découvertes et innovations financées par des capitaux publics. Cela donne souvent naissance à de petites start-up qui sont rachetées par la suite à des prix exorbitants par des grands groupes et comptabilisées comme des coûts de développement.

\section{Les prix de développement des médicaments comprennent également les énormes prix d'achat du «pipeline shopping».}

Cela fait des années que le débat fait rage sur les coûts moyens de développement; l'étude de Di Masi et al. (la plus souvent citée) estimait jusqu'à présent ces coûts entre 1,1 et 2,8 milliards de dollars (dans l'hypothèse de $11 \%$ de coûts du crédit et d'un taux de réussite clinique de 12\%) [3]. Une étude récente qui analyse 355 nouveaux médicaments homologués par la FDA entre 2009 et 2018 arrive à des estimations de coûts nettement moindres: après prise en compte des coûts des études avortées, les coûts médians d'investissement de recherche et de développement pour le lancement sur le marché d'un nouveau médicament sont évalués à 985 millions de dollars (intervalle de confiance 95\%: 684 à 1229 millions de dollars) [4]. Les entreprises pharmaceutiques investissent aujourd'hui bien plus en marketing, fabrication et distribution qu'en recherches et développement [5]. Rien qu'aux États-Unis, l'industrie pharmaceutique et des produits de santé a dépensé ces 20 dernières années 230 millions de dollars par an en lobbying, c'est-à-dire davantage que n'importe quel autre secteur [6]. Il faut noter que les prix de développement des médicaments comprennent également les énormes prix d'achat du "pipeline shopping». Il n'est donc pas étonnant que les entreprises n'aient plus grand intérêt pour un marché de grande qualité des génériques ou des biosimilaires. Le nombre de géné- 
riques essentiels qui ne sont plus disponibles augmente chaque jour, la médecine de premier recours de notre population est confrontée à un péril croissant.

\section{Qu'est-ce qui détermine le prix d'un nouveau médicament?}

Nombre de personnes ignorent le fonctionnement de la tarification pour un nouveau médicament, et aussi qu'aux États-Unis les prix des nouveaux médicaments ne sont soumis à aucune règle et sont laissés à la libre appréciation des entreprises. Toutes les compagnies pharmaceutiques essaient en conséquence d'obtenir l'homologation de leurs nouveaux médicaments aux États-Unis, car le marché américain de la santé est le plus rentable au monde et génère le plus de profits. Après l'homologation par l'autorité américaine de la santé FDA, la société fixe le prix de son médicament à sa guise. Dans ce contexte, les entreprises (sociétés "shareholder value» qui sert à générer un maximum de profits, et non le patient ou le bien commun de notre société. Une fois le prix maximum fixé, il reste protégé par nombre de règles et de brevets et n'est donc pas disponible sur le marché libre, ce qui le maintient durablement à un niveau élevé [2]. En Europe, la plupart des autorités s'orientent sur les prix américains en dépit des procédures ETS. Il n'est donc pas étonnant que l'industrie pharmaceutique compte parmi les secteurs les plus rentables.

En Suisse, il existe des brevets dans le domaine chimique depuis 1907. Ce n'est toutefois qu'en 1980, avec le BayhDole Act, que les instituts publics de recherche ont été autorisés à breveter et licencier leurs résultats et à créer ou céder des start-up à des conditions avanta-

\section{L'essentiel en bref}

- Depuis le début des années 80, le marché pharmaceutique mondial n'a cessé de croître. En 2003, le profit du secteur pharmaceutique a dépassé le profit cumulé de toutes les industries listées par Forbes.

- Le nombre de médicaments génériques essentiels qui ne sont plus disponibles augmente chaque jour, et l'approvisionnement de base est de plus en plus menacé.

Médecin responsable

Pneumologie et Allergologie, centre CF

Hôpital pédiatrique de la

Suisse orientale

Claudiusstrasse 6

CH-9006 Saint-Gall

juerg.barben[at]kispisg.ch au bien commun de la population.

- Le système de santé fondé sur la solidarité ne doit pas devenir un marché de la santé orienté uniquement sur la maximisation du profit. anonymes) doivent prendre en considération la

geuses [7]. Ceci doit permettre aux entreprises américaines davantage d'innovation et de productivité, et conserver les profits aux États-Unis. On constate qu'étonnamment, jusqu'à présent, il n'est pas prouvé que les brevets encouragent de nouvelles inventions ou même qu'ils sont indispensables pour cela [8].

\section{Conclusions}

En résumé, il faut retenir que la marge bénéficiaire de l'industrie pharmaceutique est beaucoup trop élevée par rapport à d'autres secteurs et ne reflète plus le risque d'investissement des coûts de développement. La majeure partie des coûts des médicaments innovants reflète de nos jours l'achat de start-up ainsi que le lobbying et d'autres activités de relations publiques plutôt que les coûts réels de développement. Il faut d'urgence une transparence totale de la tarification, avec des modèles de prix compréhensibles et, à chaque fois que c'est nécessaire, des licences obligatoires et l'interdiction de mesures marketing exagérées pour le lancement des médicaments onéreux. Pour cela, il faut enfin une volonté politique d'établir des règles du jeu équitables valables à l'international. "Continuer comme ça» n'est pas tenable, même dans les économies les plus riches, au plus tard depuis la crise du coronavirus. Le système de santé solidaire ne doit pas être abandonné comme c'est le cas actuellement au profit d'un marché de la santé orienté vers la maximisation des profits, où de grands groupes peuvent s'enrichir sans mesure sur le dos de personnes malades.

\section{Crédits photo}

(c) Natalyermolina2018 | Dreamstime.com

\section{Références}

1 Möhr P. Personalisierte Medizin und ihre Triebkräfte. BMS. 2016;97:1018-20.

2 Cerny T. Warum Patente in der Onkologie heute schädlich sind. Bulletin suisse du cancer. 2020;202:114-7.

3 DiMasi JA et al. Innovation in the pharmaceutical industry: New estimates of R\&D costs. J Health Econ. 2016;47:20-33.

4 Wouters $\mathrm{OJ}$ et al. Estimated Research and Development Investment Needed to Bring a New Medicine to Market. JAMA. 2020;323:844-53.

5 Tay-Teo K et al. Comparison of Sales Income and Research and Development Costs for FDA-Approved Cancer Drugs Sold by Originator Drug Companies. JAMA Netw Open. 2019;2:e186875

6 Wouters OJ. Lobbying Expenditures and Campaign Contributions by the Pharmaceutical and Health Product Industry in the US. JAMA Intern Med. 2020;180:688-97.
Markel H. Patents, Profits, and the American PeopleThe Bayh-Dole Act of 1980. N Engl J Med. 2013;369:794-6.

8 Ridley M. Rasender Fortschritt? Schön wär's. NZZ 4.6.2020. 